\title{
Renal anomalies in families of individuals with congenital solitary kidney
}

Elizabeth McPherson, MD

\begin{abstract}
Purpose: Congenital solitary kidney affects 1 in 1000 persons, whereas bilateral renal agenesis affects 1 in 5000 . Because congenital solitary kidney is increased in parents of infants with bilateral renal agenesis and fetuses with renal anomalies on ultrasound, offspring of patients with congenital solitary kidney may be at risk of both unilateral and bilateral renal anomalies. The goal was to establish empiric risk estimates for counseling individuals with congenital solitary kidney. Methods: Through a computerized review of records, subjects with congenital solitary kidney (defined as presumably congenital absence or severely reduced function of one kidney) were identified and asked to complete a questionnaire regarding urogenital anomalies in relatives. Results: Empiric risks of $7 \%$ for offspring, $4 \%$ for parents, and $2.5 \%$ for siblings are minimal because not all relatives underwent ultrasound studies. The incidence of bilateral renal agenesis in offspring of congenital solitary kidney probands is $0.8 \%$, which is much greater than the population risk but less than that for families with previous bilateral renal agenesis. Concordance for type of anomaly in affected relatives is only $50 \%$, suggesting that various anomalies included in congenital solitary kidney (agenesis, dysplasia, cystic dysplasia) may overlap. Horseshoe kidney has $80 \%$ concordance and may be a separate autosomal dominant condition. Conclusion: Offspring and other relatives of individuals with congenital solitary kidney have significantly increased renal disease. Ultrasound is recommended for first-degree relatives, including fetuses at risk. Genet Med 2007:9(5):298-302.
\end{abstract}

Key Words: Empiric risks, horseshoe kidney, renal agenesis, renal dysplasia, solitary kidney

\section{INTRODUCTION}

Because congenital solitary kidney is frequently asymptomatic, it is often discovered by chance on pre- or postnatal ultrasound or at autopsy. Estimates of the prevalence of solitary kidney approximate 1 in 1300 on ultrasound screening of healthy school-aged children ${ }^{1}$ and approximately 1 in $1000^{2}$ in autopsy series. Distinction among true renal agenesis, severe hypoplasia, and cystic dysplasia with subsequent atrophy can be difficult both on ultrasound and at autopsy. Theoretically, because renal agenesis is the result of failure of the ureteric bud to contact the metanephric blastema, the kidney and ureter should be completely absent with no rudiments detectable. This would be in contrast to atrophy of a previously hypoplastic, dysplastic, or multicystic kidney, which should leave a rudimentary kidney and ureter. In actual practice, however, ultrasound can only distinguish between renal agenesis and atrophy if previous studies show the presence of the now atro-

From the Department of Medical Genetic Services, Marshfield Clinic, Marshfield, Wisconsin. Elizabeth McPherson, MD, Department of Medical Genetic Services 3C1, Marshfield Clinic, 1000 North Oak Avenue, Marshfield, WI 54449.E-mail: mcpherson.elizabeth@marshfieldclinic.org

The author declares no conflict of interest.

Submitted for publication January 25, 2007.

Accepted for publication February 28, 2007.

DOI: 10.1097/GIM.0b013e3180544516 phic kidney, and even autopsy can sometimes fail to distinguish between these scenarios.

In one prenatal ultrasound study, unilateral renal agenesis was not identified among 12,000 fetuses screened, but 5 of $12,000(1$ in 2,200) presented prenatally with cystic dysplasia and postnatally developed unilateral severe renal hypoplasia or atrophy indistinguishable from unilateral renal agenesis. ${ }^{3} \mathrm{~A}$ more recent study of infants prenatally suspected to have unilateral renal hypoplasia showed several with progressive involution leading to unilateral renal "aplasia." ${ }^{4}$ Unilateral renal agenesis does exist prenatally, however, as documented by Bronshtein et al., ${ }^{5}$ who found 9 cases among 6350 transvaginal ultrasounds ( 1 in 705), all confirmed after delivery.

Because renal agenesis, hypoplasia, and cystic dysplasia have been reported in the same syndromes, the same families and occasionally even the same individual, they can, when unilateral, be included together under the heading of congenital solitary kidney. Horseshoe kidney and crossed fused ectopia are, in some instances, forms of solitary kidney because there is a significant incidence of dysplasia in horseshoe and ectopic kidneys. ${ }^{6}$ Furthermore, horseshoe and ectopic kidney have been reported in the same families ${ }^{7}$ and with the same other anomalies as unilateral renal agenesis.

Although most individuals with congenital solitary kidney are healthy, they are at increased risk of other anomalies, especially of the urogenital system. Early identification of these individuals can help to prevent complications. The develop- 
mental abnormality resulting in agenesis or aplasia of one kidney is not always strictly unilateral. Anomalies of the solitary kidney requiring follow-up and occasionally surgical treatment are found in $33 \%{ }^{8}$ to $48 \%{ }^{9}$ of patients with unilateral renal agenesis. Genital anomalies are found in $37 \%^{10}$ to $76 \%^{11}$ of female patients and $11.5 \%^{2}$ to $77 \%{ }^{12}$ of male patients with congenital solitary kidney. The incidence of genital anomalies among male patients might be underestimated because some studies did not report absence of the vas deferens, which is the most frequent genital anomaly in male patients with renal agenesis and can be bilateral even if the renal agenesis is unilateral. In a recent study of men with bilateral congenital absence of the vas deferens, $33 \%$ had unilateral renal agenesis. ${ }^{13}$ With the exception of consequences of oligohydramnios, the types of nonurogenital anomalies in patients with congenital solitary kidney are similar to those among patients with bilateral renal agenesis, although the frequency is less. This includes a variety of syndromes such as chromosomal anomalies, caudal dysgenesis, and VATER, as well as isolated anomalies of the cardiovascular, skeletal, and central nervous systems.

Bilateral renal agenesis is uniformly lethal, is more easily ascertained than solitary kidney, and occurs in 0.4 to 3.9 per 10,000 births. The empiric recurrence risk for perinatal lethal renal disease is $3.5 \%{ }^{14}$ to $4.4 \% .{ }^{15}$ After the birth of a child with perinatal lethal renal disease, ultrasound is usually offered in subsequent pregnancies. Congenital solitary kidney and other nonlethal urogenital anomalies are found at increased frequency among parents and other relatives of infants with bilateral renal agenesis or severe dysplasia. Hilson ${ }^{16}$ first reported unilateral and bilateral renal agenesis occurring in the same family: an infant with bilateral renal agenesis whose grandmother had cystic kidneys and whose cousin had unilateral renal agenesis. Buchta et al. ${ }^{17}$ proposed the term "hereditary renal adysplasia" to describe several families that showed both unilateral and bilateral renal agenesis or dysplasia occurring in multiple generations. More recently, Roodhooft et al. ${ }^{15}$ used ultrasound to document unilateral renal agenesis and other nonlethal urogenital anomalies in a parent of 10 of $41(24 \%)$ infants with bilateral renal agenesis or severe dysgenesis. No specific gene for hereditary renal adysplasia has been identified, although Sanna-Cherchi et al. ${ }^{18}$ recently mapped nonsyndromic renal hypodysplasia to $1 \mathrm{p} 32-33$.

Because individuals with congenital solitary kidney are usually healthy, they are seldom referred for genetic evaluation, but the observation of many families with both unilateral and bilateral renal disease suggests that the offspring of unilaterally affected patients may be at risk of more serious renal anomalies. In the studies mentioned above, ascertainment may be biased because many individuals with congenital solitary kidney have been detected only after a family member was born with bilateral renal disease. The only systematic study of relatives of individuals with nonlethal renal anomalies was provided by Bronshtein et al., ${ }^{5}$ who noted renal anomalies in a parent of 3 of 21 (14\%) fetuses with minor renal anomalies (unilateral renal agenesis, pelvic kidney, and double collecting system) on ultrasound. All studies of the genetic implications of congenital solitary kidney have been based on small numbers of families, and most are biased because of ascertainment through probands with bilateral disease. More information is needed to provide data about risks to close relatives, especially offspring of individuals with congenital solitary kidney.

\section{MATERIALS AND METHODS}

Computerized records from 1979 to the present in a large multispecialty clinic were used to identify individuals with diagnoses of aplastic kidney, atrophic kidney, cystic renal dysplasia, dysplasia kidney, horseshoe kidney, hypoplastic kidney, nonfunctioning kidney, pelvic kidney, renal anomaly, single kidney congenital (left, right, or not specified), and unilateral small kidney. The 366 charts identified were reviewed and, after exclusion of subjects who lacked clear evidence of unilateral renal disease or who had adult polycystic kidney or primarily acquired renal anomalies, 203 potential subjects with congenital solitary kidney (defined as presumably congenital absence or severely reduced function of one kidney) remained.

After approval by the institutional review board, the potential subjects, parents/guardians of pediatric or incompetent subjects, or next of kin for deceased subjects were sent a letter explaining the study, and 82 (40\%) agreed to participate. Participants (or parents for juvenile subjects and next of kin for deceased subjects) completed a questionnaire regarding pregnancy outcomes for the subject or partner, as well as urogenital anomalies and other birth defects in first- and second-degree relatives.

The renal anomalies of subjects and relatives were classified into four major clinical categories: unilateral renal agenesis, unilateral renal dysplasia/hypoplasia, cystic renal dysplasia, and horseshoe kidney. Associated anomalies, including other urogenital anomalies, were recorded for all subjects and affected relatives. One relative with uterus didelphys was counted as affected with congenital solitary kidney, although data on her renal status were lacking. Nonurogenital anomalies were simply recorded, not used to determine affected or unaffected status with respect to congenital solitary kidney. Information on pregnancy outcomes of subjects who conceived or fathered at least one pregnancy was used to calculate the rates of miscarriage and stillbirth. Information on urogenital anomalies in relatives was used to calculate empiric risk figures for different classes of relatives, as well as the concordance among relatives. The data were also analyzed by gender and type of renal anomaly of the proband.

\section{RESULTS}

Among 1,129,877 unique patients with computerized records available, only 203 were identified with congenital solitary kidney, an incidence of approximately 1 in 5500. It should be noted, however, that solitary kidney was not excluded in most of the remainder, as only a minority had renal ultrasounds or other definitive studies included in the records reviewed. 
The questionnaire was returned by $82(40 \%)$ subjects. No further review or classification was possible for those who declined participation or failed to return their questionnaires. The rate of response was similar to other questionnaire studies in the institution. There were more female than male participants (48 vs. 34), although the sex ratio was equal among clinic patients and among the 203 eligible subjects. Among participants, 40 of $82(49 \%)$ had unilateral renal agenesis, 20 of 82 (24\%) had unilateral renal dysplasia/hypoplasia, 16 of 82 (19.5\%) had horseshoe kidney, and 6 of 82 (7\%) had cystic renal dysplasia. The distribution in the total group eligible was comparable (slightly $<50 \%$ unilateral renal agenesis, nearly $25 \%$ unilateral renal dysplasia/hypoplasia, $20 \%$ horseshoe kidney, and the remaining $5 \%$ to $6 \%$ cystic renal dysplasia).

Among female subjects, 9 of 47 (19\%) had documented uterine malformations (2 Rokitansky, 1 unicornuate, 3 bicornuate, 3 didelphys). This is lower than that in most studies of solitary kidney and clearly represents a minimal estimate because most of the remainder had no data regarding uterine structure. Overall, 29 of 38 (74\%) female subjects older than 18 years had been pregnant at least once. Of the women older than 18 who had not conceived, 4 of $9(44 \%)$ had major uterine anomalies (including the 2 with absent uterus) compared with only 5 of $29(17 \%)$ of those who had been pregnant. Thus, uterine anomalies reduce but do not completely prevent fertility. Unilateral testicular agenesis was reported in 2 of $35(6 \%)$ male subjects. The subjects were not directly asked about involuntary infertility, and none of the subjects were evaluated for congenital absence of the vas deferens. It may be significant, however, that only 11 of 20 (55\%) men older than 18 years had fathered any pregnancy, and of the men who had not fathered a child, 2 of 9 had testicular anomalies. Subjects or their partners had a total of 153 pregnancies, including 125 liveborn offspring, 25 miscarriages (16\%), and 3 stillbirths (2\%).

Seven subjects (8.5\%) had additional nonurogenital anomalies. One patient had Wilms tumor, contralateral renal dysplasia, and developmental delay but had not been tested for changes in the WT1 gene. The remainder included one patient with Hirschsprung disease, one with Turner syndrome, one with Goldenhar syndrome, and three with multiple anomalies, including congenital heart disease, but not fitting any known syndrome.

Overall, 16 subjects reported a total of 25 affected relatives. Data are shown in detail in Table 1. The distribution of anomalies in the index subjects was comparable to that for all subjects (8 of 16 [50\%] unilateral renal agenesis, 4 of 16 [25\%] unilateral renal dysplasia/hypoplasia, 4 of 16 [25\%] horseshoe kidney). Empiric risk figures were calculated for first-degree relatives based on the family histories given by the subjects. Individuals with unknown urogenital status were counted as unaffected for empiric risk calculations. Among 125 offspring of affected individuals, 9 were affected with urogenital anomalies, resulting in an empiric risk of 7\%. This included 1 of 125 $(0.8 \%)$ with bilateral renal agenesis. Offspring of unilateral renal agenesis individuals considered separately were at greatest risk, with 7 of 59 (12\%) affected. The empiric risks for parents

Table 1

Renal anomalies in relatives of congenital solitary kidney probands

\begin{tabular}{|c|c|c|c|c|}
\hline & Offspring affected & Parents affected & Sibs affected & $2^{\text {nd }}$ degree affected \\
\hline URA $(n=40)$ & $7 / 59(12 \%)$ & $3 / 80(4 \%)$ & $2 / 113(2 \%)$ & \\
\hline \multirow[t]{5}{*}{ Multiplex subset, $\mathrm{n}=8(20 \%)$} & $7 / 15(47 \%)$ & $3 / 16(19 \%)$ & $2 / 28(7 \%)$ & \\
\hline & $1 \mathrm{BRA}$ & 2 URA & 2 URA (1+ MCA) & \\
\hline & 4 URA (1+ MCA) & $1 \mathrm{URD}$ & & \\
\hline & $1 \mathrm{CRD}$ & & & \\
\hline & $1 \mathrm{UT}+\mathrm{UNK}$ renal & & & \\
\hline URD/URH $(n=20)$ & $1 / 47(2 \%)$ & $1 / 40(2.5 \%)$ & $4 / 76(5 \%)$ & \\
\hline \multirow[t]{4}{*}{ Multiplex subset, $\mathrm{n}=4(20 \%)$} & $1 / 11(9 \%)$ & $1 / 8(12.5 \%)$ & $4 / 19(21 \%)$ & $2 \mathrm{CRD}$ \\
\hline & 1 UNK renal & 1 UNK renal & $2 \mathrm{URD}$ & \\
\hline & & & 1 URA & \\
\hline & & & $1 \mathrm{CRD}$ & \\
\hline $\operatorname{HSK}(n=16)$ & $1 / 19(5 \%)$ & $3 / 32(10 \%)$ & $0 / 46$ & \\
\hline \multirow[t]{2}{*}{ Multiplex subset, $\mathrm{n}=4(25 \%)$} & $1 / 19(5 \%)$ & $3 / 8(37.5 \%)$ & $0 / 10$ & $1 \mathrm{HSK}$ \\
\hline & 1 URA & 3 HSK & & \\
\hline $\operatorname{CRD}(n=6)$ & 0 & 0 & $\mathbf{0}$ & \\
\hline Multiplex subset, $\mathrm{n}=0$ & 0 & 0 & 0 & \\
\hline Probands $(n=82)$ & 9/125 (7\%) & 7/164 (4\%) & $6 / 245(2.5 \%)$ & $3 / ?$ \\
\hline
\end{tabular}

BRA, bilateral renal agenesis; CRD, cystic renal dysplasia; MCA, multiple congenital anomalies; URA, unilateral renal agenesis; URD, unilateral renal dysplasia; URH, unilateral renal hypoplasia; UNK, specific anomaly unknown; UT, uterine. 
and siblings were $4 \%$ (7 of 164 affected) and 2.5\% (6 of 245 affected), with no known cases of bilateral renal agenesis in this group, although there were several neonatal deaths of unknown cause. Ascertainment for renal anomalies in parents was presumably less complete than in offspring, because $28 \%$ of subjects indicated uncertainty about the renal status of parents, whereas only $7 \%$ indicated uncertainty about renal anomalies in their offspring.

Concordance for type of renal anomaly (unilateral/bilateral renal agenesis, unilateral renal dysplasia/hypoplasia, horseshoe kidney, and cystic renal dysplasia) was 52\% (13 of 25) overall, $58 \%$ ( 7 of 12) for unilateral renal agenesis probands, $25 \%$ ( 2 of 8) for unilateral renal dysplasia/hypoplasia probands, and $80 \%$ (4 of 5) for horseshoe kidney probands, suggesting that horseshoe kidney, at least in some cases, may be a separate genetic disorder. The frequency of associated nonurogenital anomalies in affected relatives was $12 \%$ ( 3 of 25 ), which is comparable to that among the probands.

\section{DISCUSSION}

The major strength of this study is the ability to screen large numbers of medical records and to obtain histories for large numbers of families so that, despite incomplete ascertainment, more individuals and families with congenital solitary kidney were identified compared to ultrasound based studies. Family history data were obtained for 82 probands, identifying 25 additional affected relatives in 16 families, whereas Bronshtein et al. ${ }^{5}$ studied 21 probands with nonlethal renal disease identifying three additional affected relatives in three families. Roodhooft et al. ${ }^{15}$ found nonlethal urogenital anomalies in relatives of 13 of 41 bilateral renal agenesis probands and bilateral renal agenesis in 2 of 127 siblings of bilateral renal agenesis patients.

Another strength of the study is the inclusion of individuals with various forms of solitary kidney. This was essential as an ultrasound study in living individuals cannot always distinguish atrophy of a dysplastic or cystic kidney from primary agenesis. Furthermore, it allowed identification of families in which affected relatives might have different types of renal disease, such as the previously described hereditary renal adysplasia, in which renal agenesis, hypoplasia, dysplasia, and cystic dysplasia have all been reported. ${ }^{17-20}$ The observation that concordance for the precise type of renal anomaly was only approximately $50 \%$ among relatives suggests that many families would have been missed if the study had been limited to renal agenesis.

A weakness of the study is under-ascertainment because of dependence on existing medical records and proband knowledge of their relative's health histories. The incidence of congenital solitary kidney detected by a computer-based search of clinic records is much less than that in ultrasound or autopsybased studies and represents an underestimate of the true incidence of congenital solitary kidney in the population. A minority of individuals in the population have had renal ultrasound. Furthermore, not all diagnoses made elsewhere are included in the computerized records available for review. The questionnaire format would be expected to result in incomplete ascertainment of affected relatives because asymptomatic relatives may not have had ultrasound; furthermore, individuals completing the questionnaire may not have been aware of all diagnoses that had been made in their relatives.

The major goal of the study was development of empiric risk estimates for use in genetic counseling of individuals with congenital solitary kidney. Risks for both unilateral and bilateral renal anomalies in offspring and other relatives of individuals with congenital solitary kidney were concerns. There is only one previous study of urogenital anomalies in parents of fetuses with nonlethal renal anomalies, primarily congenital solitary kidney, ${ }^{5}$ and no previous published empiric risks to offspring or siblings of individuals with congenital solitary kidney. The empiric risk of $7 \%$ can be compared with the smaller ultrasound-based Bronshtein study, which should have resulted in nearly complete ascertainment. In that study, renal anomalies were identified in one parent of $14 \%$ (3 of 21) of unilaterally affected fetuses. Because both parents of each fetus were studied, this means $7 \%$ of parents were affected. If $14 \%$ of cases were the result of autosomal dominant inheritance from an affected parent, the risk for siblings and offspring should also be approximately $7 \%$. Although the estimate of recurrence risk to offspring must be regarded as minimal because of the potential for under-ascertainment of asymptomatic but affected relatives, the agreement with Bronshtein's data suggests that it is clinically meaningful. The empiric risks for other relatives are lower than expected and may represent more significant underestimates because many probands in this study indicated uncertainty about the health of their parents and siblings.

In this study, there was only one case of bilateral renal agenesis among 125 offspring of individuals with congenital solitary kidney. This gives a risk of $0.8 \%$, which can be compared with the $1.8 \%$ risk that might have been predicted by applying Bayes' theorem to the data of Roodhooft et al. ${ }^{15}$ The estimate is minimal because additional fetuses with bilateral renal agenesis could have been missed among miscarriages or stillbirths. In this study, the miscarriage rate was similar to that of the general population, indicating that selective miscarriage of affected fetuses is not likely to bias the results. Although the stillbirth rate was double that of the general population, the difference was not significant because of small numbers. It is possible that a few cases of bilateral renal agenesis could have been missed because none of the three stillbirths were autopsied. Considering these results and those of Roodhooft et al ${ }^{15}$ the risk of bilateral renal agenesis among offspring of individuals with congenital solitary kidney seems to be $1 \%$ to $2 \%$, which is significantly greater than the general population risk of 1 in 2,500 to 1 in 25,000; therefore, ultrasound should be offered in pregnancies of individuals with congenital solitary kidney.

A secondary goal was identification of multiplex families. The largest family identified in the study contains a proband with unilateral renal dysplasia; a grandparent, sibling, and niece with cystic 
renal dysplasia; a sibling with unilateral renal agenesis; and an offspring with incompletely diagnosed renal disease, suggesting that the underlying condition might be cystic renal dysplasia with some individuals progressing to severe dysplasia or atrophy indistinguishable from unilateral renal agenesis. In addition to this family, two other families who seemed to have hereditary renal adysplasia were identified in the study group (one with four cases of unilateral renal agenesis and one case of bilateral renal agenesis in three generations, and a smaller family with three cases of unilateral renal agenesis in two generations). There were also six parent/child pairs and three sibling pairs with unilateral renal agenesis and/or unilateral renal dysplasia. It is tempting to speculate that most of the familial recurrences of unilateral renal agenesis/unilateral renal dysplasia might be the result of hereditary renal adysplasia. The occurrence of bilateral renal agenesis in these families, however, is much less than expected given previous estimates of the penetrance for bilateral renal agenesis and unilateral renal agenesis, each approximately 33\% in hereditary renal adysplasia heterozygotes..$^{19}$ Either previous estimates are biased because of ascertainment of hereditary renal adysplasia through bilaterally affected probands, or a significant number of unilateral renal agenesis recurrences are the result of some other cause. In any case, it is reassuring for counseling purposes that the empiric risk for bilateral renal agenesis in offspring of congenital solitary kidney probands is only approximately $1 \%$, rather than the $15 \%$ to $20 \%$ predicted for hereditary renal adysplasia heterozygotes.

The decision to include horseshoe kidney was fortuitous, because it led to the unanticipated finding of three families in which horseshoe kidney recurred in two generations, suggesting that horseshoe kidney may occur as a previously undescribed autosomal dominant condition. The high concordance $(80 \%)$ for horseshoe kidney in families with a horseshoe kidney proband supports this hypothesis. Linkage analysis for horseshoe kidney, however, is not possible, because the total numbers are small and the largest family only contains three affected individuals.

Although the pedigrees mentioned above strongly support autosomal dominant inheritance, recurrence risks are lower than expected for a uniformly autosomal dominant condition. Heterogeneity, incomplete penetrance, or incomplete ascertainment are possible explanations. Heterogeneity is documented because at least two different autosomal dominant genes seem to be involved (hereditary renal adysplasia and horseshoe kidney). Other Mendelian patterns are less likely because 13 of 16 families demonstrate vertical transmission. Multifactorial causation cannot be excluded but is unlikely to account for all the observed recurrences because these data and that of Bronshtein et al. ${ }^{5}$ show risks to parents and offspring that are higher than expected for a strict multifactorial condition, exceeding the square root of the incidence in the population.

Among 82 probands with congenital solitary kidney, a questionnaire method was used to identify 25 affected relatives in
16 families. This study demonstrates genetic heterogeneity with at least two different autosomal dominant conditions (hereditary renal adysplasia and nonsyndromic horseshoe kidney) occurring in the study group. Most of the families, however, are too small for recognition of a specific genetic pattern. Minimal empiric risks to offspring of individuals with congenital solitary kidney are $7 \%$ congenital solitary kidney and $1 \%$ for bilateral renal agenesis. Ultrasound is recommended to check for urogenital anomalies in first-degree relatives of persons found to have congenital solitary kidney. Prenatal diagnosis by ultrasound is recommended for pregnancies in which the patient or her partner has congenital solitary kidney.

\section{ACKNOWLEDGMENTS}

The author thanks Marshfield Clinic Research Foundation for its support through the assistance of Linda Weis and Alice Stargardt in the preparation of this manuscript.

\section{References}

1. Sheih CP, Liu MB, Hung CS, Yang KH, et al. Renal abnormalities in schoolchildren Pediatrics 1989;84:1086-1090.

2. Doroshow LW, Abeshouse BS. Congenital unilateral solitary kidney: report of 37 cases and a review of the literature. Urol Surv 1961;11:219-229.

3. Helin I, Persson PH. Prenatal diagnosis of urinary tract abnormalities by ultrasound. Pediatrics 1986;78:879-883

4. Hiraoka M, Tsukahara H, Ohshima Y, Kasuga K, et al. Renal aplasia is the predominant cause of congenital solitary kidneys. Kidney Int 2002;61:1840-1844.

5. Bronshtein M, Bar-Hava I, Lightman A. The significance of early second-trimester sonographic detection of minor fetal renal anomalies. Prenat Diagn 1995;15:627632.

6. Borer JG, Glassberg KI, Kassner EG, Schulsinger DA, et al. Unilateral multicystic dysplasia in 1 component of a horseshoe kidney: case reports and review of the literature. J Urol 1994;152:1568-1571.

7. Schwyzer U, Litschgi M, Schinzel A. [Familial kidney agenesis: renal adysplasia as a cause of urogenital anomalies in 3 generations]. Geburtshilfe Frauenheilkd 1989;49: 759-761.

8. Krzemien G, Roszkowska-Blaim M, Kostro I, Wojnar J, et al. Urological anomalies in children with renal agenesis or multicystic dysplastic kidney. J Appl Genet 2006; 47:171-176.

9. Cascio S, Paran S, Puri P. Associated urological anomalies in children with unilateral renal agenesis. J Urol 1999;162:1081-1083.

10. Thompson DP, Lynn HB. Genital anomalies associated with solitary kidney. Mayo Clin Proc 1966;41:538-548.

11. Collins DC. Congenital unilateral renal agenesis. Ann Surg 1932;95:715-726.

12. Engel D. Ueber eine seltene form der urogenitalmissbildung. Beitr Path Anat Allg Path 1920;lxvii:549-558.

13. Kolettis PN, Sandlow JI. Clinical and genetic features of patients with congenita unilateral absence of the vas deferens. Urology 2002;60:1073-1076.

14. Carter CO, Evans K, Pescia G. A family study of renal agenesis. J Med Genet 1979; 16:176-188.

15. Roodhooft AM, Birnholz JC, Holmes LB. Familial nature of congenital absence and severe dysgenesis of both kidneys. N Engl J Med 1984;310:1341-1345.

16. Hilson D. Malformation of ears as sign of malformation of genito-urinary tract. $B M J$ 1957;2:785-789.

17. Buchta RM, Viseskul C, Gilbert EF, Sarto GE, et al. Familial bilateral renal agenesis and hereditary renal adysplasia. Z Kinderheilkd 1973;115:111-129.

18. Sanna-Cherchi S, Caridi G, Weng PL, Dagnino M, et al. Localization of a gene for non-syndromic renal hypodysplasia to chromosome 1p32-33. Am J Hum Genet 2007;80:539-549.

19. McPherson E, Carey J, Kramer A, Hall JG, et al. Dominantly inherited renal adysplasia. Am J Med Genet 1987;26:863-872.

20. Doray B, Gasser B, Reinartz I, Stoll C. Hereditary renal adysplasia in a three generations family. Genet Couns 1999;10:251-257. 